\title{
O impacto da Medicina Narrativa na formação médica:
}

\author{
uma revisão bibliográfica
}

\section{The impact of Narrative Medicine in medical training: a literature review}

\section{El impacto de la Medicina Narrativa en la formación médica: una revisión de la literatura}

\author{
Fátima Geovanini ${ }^{1}$ \\ Luiza Otero ${ }^{2}$ \\ Susana Teixeira ${ }^{3}$
}

\begin{abstract}
Resumo: Este estudo apresenta uma revisão integrativa da literatura sobre avaliação e validação dos resultados da Medicina Narrativa - MN. O objetivo foi identificar as atividades empregadas no campo da MN, o público-alvo, os modelos metodológicos mais utilizados para avaliação e os resultados alcançados. O levantamento inicial gerou 808 artigos. Com os critérios estabelecidos, 693 artigos foram excluídos e 115 indicados para análise e discussão. Ao final 15 artigos, exclusivamente de pesquisa, foram selecionados e analisados quanto aos objetivos propostos. Dentre os resultados identificados: 1- a MN apresenta impactos positivos; 2- o modelo qualitativo tem sido o mais empregado para avaliação dos resultados; 3- a manutenção dos benefícios adquiridos pode estar atrelada à continuidade do trabalho; 4- a junção da teoria com a prática pode potencializar os resultados adquiridos. Os estudos sugerem que novas pesquisas devem ser feitas no campo da MN.
\end{abstract}

Palavras-chave: narrativas, medicina narrativa, humanidades médicas.

Summary: This study consists of an integrative literature review on the assessment and validation of the results of Medicine Narrative. The aim of literature review is to identify the activities used in the field of NM, the target audience of the proposed activities, the methodological models most commonly used for evaluation and the results achieved by them. The initial survey generated 808 articles. Based on the established criteria, 693 articles were excluded and 115 were selected for analysis and discussion among the researchers. At the end, 15 articles, exclusively research articles, were selected and analyzed according to the proposed objectives. Among the results identified we can highlight that 1- the MN presents positive impacts; 2 - the qualitative model has been the most used for evaluation of the results; 3 - the maintenance of the acquired benefits may be linked to the continuity of the work; 4- the junction of theory with practice can enhance the acquired results. All studies suggest that new research must be done in the field of NM.

Keywords: narratives, narrative medicine, medical humanities

Resumen: El presente estudio consiste en una revisión bibliográfica integradora sobre la evaluación y validación de los resultados de la Medicina Narrativa. El objetivo de la revisión fue identificar las actividades utilizadas en el ámbito de la NM, el público objetivo de las actividades propuestas, los modelos metodológicos más utilizados para la evaluación y los resultados obtenidos por ellos. La encuesta inicial generó 808 artículos. Según los criterios establecidos, se excluyeron 693 artículos y se seleccionaron 115 para su análisis y discusión entre los investigadores. Al final, se seleccionaron 15 artículos, exclusivamente de investigación, y se analizaron de acuerdo con los objetivos propuestos. Entre los resultados identificados podemos destacar que: 1- el MN presenta impactos positivos; 2- el modelo cualitativo ha sido el más utilizado para la evaluación de los resultados; 3- el mantenimiento de los beneficios adquiridos puede estar ligado a la continuidad del trabajo; 4- la unión de la teoría con la práctica puede potenciar los resultados adquiridos. Todos los estudios sugieren que deben realizarse nuevas investigaciones en el campo de la NM.

Palabras clave: narrativas, medicina narrativa, humanidades médicas.

1 Faculdade de Medicina da Universidade Estácio de Sá 2 Faculdade de Medicina da Universidade Estácio de Sá 3 Universidade do Porto, Universidade Fernando Pessoa, Universidade Católica Portuguesa; Faculdade de Medicina da Universidade do Porto 


\section{Introdução}

O crescimento exponencial da Medicina Narrativa é notável em diversos países da Europa, Ásia e América Latina, desde a sua criação na virada do século XXI, pela médica e literata americana Rita Charon. Charon desenvolveu uma prática que, voltada inicialmente para a formação médica e, posteriormente estendida a todos os profissionais da área da saúde, busca minimizar a hiância observada entre o profissional e o paciente, ou ainda, entre o que é ensinado e o que é exigido na dinâmica relacional que se estabelece na prática clínica. Para a autora, a MN é uma prática exercida com competência narrativa, constituída por um conjunto de habilidades voltadas para reconhecer, absorver, interpretar e agir a partir das histórias contadas pelos doentes.

Embora a relação da literatura com a medicina seja antiga, a criação da MN como área do conhecimento interdisciplinar que integra a literatura, a produção narrativa e outras linguagens artísticas na sua metodologia constitui algo inovador. Nos workshops organizados por Charon e seus colaboradores já participaram, desde 2006, mais de 2000 participantes. Em 2009 foi criado o mestrado em MN da Universidade de Columbia. Desde então, a MN vem sendo utilizada em diversas universidades e cenários clínicos, tendo três linhas de atuação: educação, assistência e pesquisa, cujo foco principal é promover o cuidado com o paciente, familiares e profissionais. ${ }^{4}$

Atualmente, após vinte anos de prática, crescem as discussões em torno dos possíveis efeitos e impactos da MN na formação dos profissionais da saúde, bem como sobre o desenho metodológico mais adequado à validação de seus resultados. Esses questionamentos motivaram a elaboração desta pesquisa, impulsionada a partir de três perguntas principais: 1- Qual é o impacto da MN na formação do estudante de medicina? 2- Qual é a modalidade de pesquisa - qualitativa ou quantitativa - mais adequada à validação de seus resultados? 3- Será que o impacto da MN precisa ser validado através de pesquisa científica?

\section{Objetivos}

Identificar os modelos metodológicos utilizados para avaliar e validar o impacto das práticas de MN na educação médica e discutir a pertinência dos modelos metodológicos de pesquisa empregados em MN.

\section{Percurso metodológico}

4 Rita Charon. The Principles And Practice Of Narrative Medicine. 1st ed. Oxford: Oxford University Press, 2016 
Para o alcance dos objetivos propostos optou-se pela realização de uma revisão integrativa da literatura consistindo, a partir do delineamento do tema, no cumprimento das seguintes etapas: definição dos critérios de inclusão e exclusão; identificação dos estudos pré-selecionados e selecionados; categorização do estudo; e análise dos resultados, conforme descritos detalhadamente a seguir.

A revisão integrativa da literatura foi a metodologia eleita para a pesquisa por ser definida como um método capaz de apresentar resultados de uma pesquisa de forma objetiva, sintetizada e ordenada, proporcionando um acesso rápido aos resultados mais relevantes de um determinado tema pesquisado contribuindo, dessa forma, para o aprofundamento teórico do tema selecionado. ${ }^{5}$

O levantamento bibliográfico foi realizado entre agosto e dezembro de 2020, nas plataformas Portal Regional da BVS, SciELO, Periódicos CAPES e PubMed. Foram utilizados os seguintes descritores: medicina narrativa, educação médica, narrative medicine e medical education, em artigos publicados em português, inglês, francês e espanhol, entre os anos de 2010 e 2020.

Foram estabelecidos os seguintes critérios de exclusão: artigos em duplicidade; publicados em período anterior ao ano de 2010; em língua estrangeira fora das estabelecidas; os que não estavam disponíveis na íntegra e os que, embora apresentassem os descritores compatíveis com a busca, fugiam ao tema proposto sendo, em sua maioria, narrativas de experiências individuais.

Devido à enorme quantidade de artigos encontrados, em inglês e português, no periódico CAPES - aproximadamente 25.000 - optou-se, nesta plataforma, pela utilização exclusiva dos descritores em língua portuguesa. Todos os artigos encontrados foram inseridos e catalogados através do programa Mendeley, desenvolvido para gestão de referências bibliográficas que permite adicionar, buscar, organizar e compartilhar artigos científicos. Através deste programa todos os artigos foram lidos, selecionados e fichados.

O levantamento inicial gerou 808 artigos: 334 da BVS, 180 do PubMed, 189 da Scielo e 105 do Periódicos CAPES. Neste primeiro levantamento foram descartados 693 artigos, de acordo com os critérios de exclusão estabelecidos. Todos os 115 artigos selecionados foram inseridos em uma pasta e renomeados de forma numérica, sendo lidos, fichados e discutidos entre os pesquisadores através de suas catalogações ordinais. Destes, foram encontrados 44 artigos exclusivamente teóricos e 71 com relatos de atividades práticas, com ou sem pesquisas de campo. A revisão destes 71 artigos pretendeu identificar a pesquisa descrita, analisar os seus objetivos, o público-alvo, o método utilizado para avaliação e validação dos resultados e as conclusões apresentadas. Para análise e discussão

5 Dsar Vosgerau, Romanowski, Joana Paulin. Estudos de revisão: implicações conceituais e metodológicas. Revista de Diálogo Educacional, 41, 14 (2014): 65-189. 
neste estudo foram privilegiados 15 artigos que apresentaram metodologia consistente e clareza em sua estruturação e avaliação dos resultados, seja por método qualitativo ou quantitativo. As 56 publicações restantes, por não se tratarem de pesquisa, foram utilizadas para fundamentação teórica deste artigo.

\section{Resultado}

Os 15 artigos selecionados foram catalogados de acordo com as seguintes informações: ano de publicação, país de origem, tipo de atividade realizada, objetivo da pesquisa, sujeitos participantes, metodologia, instrumentos utilizados para avaliação dos dados e resultados apresentados, conforme demonstrados nas tabelas abaixo.

\section{Ano de Publicação}

\begin{tabular}{|c|c|}
\hline ANO & ARTIGOS \\
\hline 2010 & 1 \\
\hline 2012 & 2 \\
\hline 2013 & 2 \\
\hline 2014 & 1 \\
\hline 2016 & 1 \\
\hline 2017 & 1 \\
\hline 2018 & 5 \\
\hline 2019 & 1 \\
\hline 2020 & 1 \\
\hline Total & 15 \\
\hline
\end{tabular}

País de Origem

\begin{tabular}{|c|c|}
\hline PAÍS & ARTIGOS \\
\hline EUA & 5 \\
\hline BRASIL & 4 \\
\hline CHINA & 2 \\
\hline FRANÇA & 1 \\
\hline REINO UNIDO & 1 \\
\hline IRÃ & 1 \\
\hline MULTICÊNTRICO & 1 \\
\hline TOTAL & 15 \\
\hline
\end{tabular}

Atividade Realizada 
Neste aspecto optou-se por dividir os artigos em dois grupos, considerando os que realizaram todas as etapas de uma oficina de Medicina Narrativa, a saber, leitura cerrada, produção narrativa e discussão, e os que fizeram apenas uma, ou duas, dessas atividades.

\begin{tabular}{|c|c|}
\hline ATIVIDADE & ARTIGOS \\
\hline MN Completa & 10 \\
\hline MN Incompleta & 5 \\
\hline Total & 15 \\
\hline
\end{tabular}

\section{Objetivos da Pesquisa}

Todos os artigos selecionados tiveram como objetivo principal validar o impacto da Medicina Narrativa, mais especificamente, identificar o seu impacto com relação aos seguintes temas: empatia; burnout; relação médico-paciente; relações interprofissionais; trabalho em equipe; habilidades de escuta, negociação e tomada de decisão; segurança do paciente; aumento do autoconhecimento; desenvolvimento de competências afetivas; preparar o estudante para a entrada no internato; e identificar o tipo de programa de MN mais eficaz a ser aplicado.

\section{Metodologia}

\begin{tabular}{|c|c|}
\hline METODOLOGIA & ARTIGOS \\
\hline Qualitativa & 10 \\
\hline Quantitativa & 4 \\
\hline Qualitativa-Quantitativa & 1 \\
\hline Total & 15 \\
\hline
\end{tabular}

\section{Instrumentos para Avaliação dos Dados}

\begin{tabular}{|c|c|}
\hline INSTRUMENTO & ARTIGOS \\
\hline Questionário & 2 \\
\hline Narrativa & 2 \\
\hline Questionário e Narrativa & 1 \\
\hline Questionário, Narrativa, Grupo Focal & 1 \\
\hline Escala de Empatia - JSPE & 2 \\
\hline Escala de Reflexão - Reflect & 1 \\
\hline Narrativa e Prova & 1 \\
\hline Questionário e Depoimentos & 1 \\
\hline Questionário e Entrevista & 2 \\
\hline
\end{tabular}




\begin{tabular}{c|c} 
Entrevista, Observação do Participante e & 1 \\
Diário de Campo & 1 \\
Relatos & 15
\end{tabular}

\section{Dados Gerais}

\begin{tabular}{|c|c|c|c|c|c|c|c|}
\hline $\mathbf{N}^{\circ}$ & ARTIGO & ANO & LÍNGUA & PAÍS & $\begin{array}{l}\text { PARTICIPAN- } \\
\text { TES }\end{array}$ & $\begin{array}{l}\text { METODOLO- } \\
\text { GIA }\end{array}$ & AVALIAÇÃO \\
\hline 1 & $\begin{array}{l}\text { Can literature enhance on- } \\
\text { cology training? A pilot hu- } \\
\text { manities curriculum }\end{array}$ & 2010 & Inglês & EUA & $\begin{array}{l}11 \text { Residentes de } \\
\text { oncologia, oncolo- } \\
\text { gia pediátrica, ra- } \\
\text { diologia oncoló- } \\
\text { gica e neuro-onco- } \\
\text { logia. }\end{array}$ & Qualitativa & Questionário \\
\hline 2 & $\begin{array}{l}\text { L'enseignement de la méde- } \\
\text { cine narrative peut-il être } \\
\text { une réponse à l'attente de } \\
\text { formation des étudiants à la } \\
\text { relation médecin-malade? }\end{array}$ & 2012 & Francês & França & $\begin{array}{l}40 \text { Estudantes de } \\
\text { medicina }\end{array}$ & Qualitativa & $\begin{array}{c}\text { Questionários e } \\
\text { narrativas }\end{array}$ \\
\hline 3 & $\begin{array}{c}\text { Does narrative medicine } \\
\text { education improve nursing } \\
\text { students' empathic abilities } \\
\text { and academic achievement? } \\
\text { A randomised controlled } \\
\text { trial }\end{array}$ & 2018 & Inglês & China & $\begin{array}{l}180 \text { Estudantes do } \\
3^{\circ} \text { ano de enfer- } \\
\text { magem }\end{array}$ & $\begin{array}{l}\text { Quantitativa e lon- } \\
\text { gitudinal }\end{array}$ & $\begin{array}{c}\text { Escala de Empa- } \\
\text { tia } \\
\text { JSE- HP }\end{array}$ \\
\hline 4 & $\begin{array}{c}\text { Implementing a narrative } \\
\text { medicine curriculum during } \\
\text { the internship year: an inter- } \\
\text { nal medicine residency pro- } \\
\text { gram experience }\end{array}$ & 2018 & Inglês & EUA & $\begin{array}{l}13 \text { Residentes em } \\
\text { medicina interna }\end{array}$ & Qualitativa & $\begin{array}{l}\text { Questionário e } \\
\text { depoimentos }\end{array}$ \\
\hline 5 & $\begin{array}{l}\text { Implementing an interpro- } \\
\text { fessional narrative medicine } \\
\text { program in academic cli- } \\
\text { nics: Feasibility and pro- } \\
\text { gram evaluation }\end{array}$ & 2019 & Inglês & EUA & $\begin{array}{l}104 \text { Médicos e en- } \\
\text { fermeiros }\end{array}$ & $\begin{array}{l}\text { Qualitativo } \\
\text { longitudinal }\end{array}$ & $\begin{array}{l}\text { Questionário e } \\
\text { entrevista }\end{array}$ \\
\hline 6 & $\begin{array}{l}\text { Patients as teachers: a ran- } \\
\text { domised controlled trial on } \\
\text { the use of personal stories } \\
\text { of harm to raise awareness } \\
\text { of patient safety for doctors } \\
\text { in training }\end{array}$ & 2014 & Inglês & Inglaterra & $\begin{array}{l}283 \text { Médicos em } \\
\text { treinamento para } \\
\text { segurança do paci- } \\
\text { ente }\end{array}$ & $\begin{array}{c}\text { Quantitativo, caso- } \\
\text { controle (APSQ e } \\
\text { PANAS) }\end{array}$ & Questionário \\
\hline 7 & $\begin{array}{l}\text { The writing of narratives } \\
\text { and the development of col- } \\
\text { laborative practices for te- } \\
\text { amwork }\end{array}$ & 2016 & Inglês & Brasil & $\begin{array}{l}146 \text { profissionais } \\
\text { de educação física, } \\
\text { fisioterapia, psico- } \\
\text { logia, nutrição, as- } \\
\text { sistência social e } \\
\text { terapia ocupacio- } \\
\text { nal. }\end{array}$ & $\begin{array}{l}\text { Qualitativa explo- } \\
\text { ratória descritiva }\end{array}$ & $\begin{array}{l}\text { Questionário e } \\
\text { entrevista }\end{array}$ \\
\hline 8 & $\begin{array}{l}\text { Uma experiência do uso de } \\
\text { narrativas na formação de } \\
\text { estudantes de Medicina }\end{array}$ & 2018 & Português & Brasil & $\begin{array}{l}80 \text { Estudantes de } \\
\text { medicina do } 5^{\circ} \text { pe- } \\
\text { ríodo em uma dis- } \\
\text { ciplina entre } 2012 \\
\text { e } 2015\end{array}$ & Qualitativa & Narrativas \\
\hline
\end{tabular}




\begin{tabular}{|c|c|c|c|c|c|c|c|}
\hline 9 & $\begin{array}{c}\text { Avaliação do desenvolvi- } \\
\text { mento de competências afe- } \\
\text { tivas e empáticas do futuro } \\
\text { médico }\end{array}$ & 2013 & Português & Brasil & $\begin{array}{l}39 \text { Estudantes de } \\
\text { medicina }\end{array}$ & $\begin{array}{l}\text { Qualitativa explo- } \\
\text { ratória transversal }\end{array}$ & Narrativas \\
\hline 10 & $\begin{array}{l}\text { Impact of a narrative medi- } \\
\text { cine programme on heal- } \\
\text { thcare providers' empathy } \\
\text { scores over time }\end{array}$ & 2017 & Inglês & China & $\begin{array}{l}142 \text { Profissionais } \\
\text { da saúde }\end{array}$ & $\begin{array}{l}\text { Quantitativa } \\
\text { (Jefferson Scale of } \\
\text { Medicine) }\end{array}$ & $\begin{array}{c}\text { Escala de Empa- } \\
\text { tia } \\
\text { JSE- HP }\end{array}$ \\
\hline 11 & $\begin{array}{l}\text { Impact of a narrative medi- } \\
\text { cine program on reflective } \\
\text { capacity and empathy of } \\
\text { medical students in Iran }\end{array}$ & 2020 & Inglês & Irã & $\begin{array}{l}135 \text { Internos de } \\
\text { medicina }\end{array}$ & Quantitativa & $\begin{array}{c}\text { Escala } \\
\text { Reflect } \\
\text { Escala de Empa- } \\
\text { tia } \\
\text { JSPE }\end{array}$ \\
\hline 12 & $\begin{array}{l}\text { Narrativas de estudantes de } \\
\text { Medicina e Enfermagem: } \\
\text { currículo oculto e desuma- } \\
\text { nização em saúde }\end{array}$ & 2018 & Português & Brasil & $\begin{array}{l}25 \text { Estudantes de } \\
\text { medicina e enfer- } \\
\text { magem do } 2^{\circ} \text { e } 3^{\circ} \\
\text { ano }\end{array}$ & $\begin{array}{l}\text { Qualitativa (feno- } \\
\text { menologia herme- } \\
\text { nêutica) }\end{array}$ & $\begin{array}{l}\text { Entrevista, ob- } \\
\text { servação partici- } \\
\text { pante e diário de } \\
\text { campo }\end{array}$ \\
\hline 13 & $\begin{array}{l}\text { Narrative medicine as a me- } \\
\text { ans of training medical stu- } \\
\text { dents toward residency } \\
\text { competencies }\end{array}$ & 2012 & Inglês & EUA & $\begin{array}{l}12 \text { Estudantes de } \\
\text { medicina do } 4^{\circ} \\
\text { ano }\end{array}$ & $\begin{array}{l}\text { Qualitativa e lon- } \\
\text { gitudinal }\end{array}$ & $\begin{array}{l}\text { Narrativas, } \\
\text { questionário e } \\
\text { grupo focal }\end{array}$ \\
\hline 14 & $\begin{array}{c}\text { Navigating uncertainty: } \\
\text { Narrative medicine in preg- } \\
\text { nancy options counseling } \\
\text { education }\end{array}$ & 2018 & Inglês & EUA & $\begin{array}{l}103 \text { Estudantes de } \\
\text { medicina }\end{array}$ & $\begin{array}{l}\text { Quantitativo e } \\
\text { Qualitativo } \\
\text { (OSCE) }\end{array}$ & $\begin{array}{c}\text { Narrativa e } \\
\text { prova }\end{array}$ \\
\hline 15 & $\begin{array}{l}\text { The use of abstract pain- } \\
\text { tings and narratives to fos- } \\
\text { ter reflective capacity in } \\
\text { medical educators: a multi- } \\
\text { national faculty deve- } \\
\text { lopment workshop }\end{array}$ & 2013 & Inglês & $\begin{array}{l}\text { Áustria, Ir- } \\
\text { landa, Is- } \\
\text { rael, Países } \\
\text { Baixos, } \\
\text { Nova Ze- } \\
\text { lândia, No- } \\
\text { ruega, Ro- } \\
\text { mênia, Sin- } \\
\text { gapura, Es- } \\
\text { panha, In- } \\
\text { glaterra }\end{array}$ & 23 Médicos & Qualitativa & Relatos \\
\hline
\end{tabular}

\section{Quanto aos Resultados Apresentados}

Todos os artigos analisados apresentaram resultados que reafirmam o impacto positivo da Medicina Narrativa. Dentre os efeitos identificados, nos vários estudos analisados, foram apontados aumento no nível da empatia, da compaixão e do autoconhecimento; promoção das habilidades de comunicação, escuta e negociação; melhora nas relações interpessoais e redução dos níveis hierárquicos entre as equipes; incremento da postura autorreflexiva e do engajamento emocional; além da identificação de pontuações mais altas nas avaliações de desempenho clínico e técnico globais. 
Foi identificada a importância da continuidade das atividades práticas para a manutenção das habilidades conquistadas. Um estudo observou melhores resultados quando as intervenções foram complementadas com o estudo teórico da Medicina Narrativa e quando o participante demonstrou interesse em participar das atividades propostas.

\section{Discussão}

A análise das publicações permitiu observar que as falhas na graduação médica, no que tange ao ensino das humanidades médicas, é identificada em diversos países, ainda que estes apresentem significativas diferenças socioculturais. Consideramos esse um ponto relevante na medida em que, dificuldades na formação profissional do médico estão relacionadas, de fato, à origem da Medicina Narrativa e, provavelmente, à sua expansão nos últimos dez anos.

Dentre os artigos selecionados, EUA e Brasil foram os países que se destacaram em termos quantitativos de publicações. Esses dados são compatíveis com que se observa na prática uma vez que a MN nos EUA, além de ter como precursora a médica Rita Charon da Universidade de Columbia, apresenta forte influência na formação de profissionais da saúde, através de seus workshops. Por outro lado, observamos que o Brasil vem se destacando, através de estudos, atividades práticas e disciplinas ministradas na graduação médica, realizando atividades em universidades e hospitais, em cidades como o Rio de Janeiro, São Paulo e Porto Alegre, dentre outras. A MN no Brasil tem recebido forte contribuição de Portugal, através das parcerias realizadas entre os pesquisadores desses dois países, principalmente pelos programas SHARE - Universidade de Lisboa, e GERMEN - Universidade Católica Portuguesa, Universidade Fernando Pessoa, Faculdade de Medicina da Universidade do Porto e Instituto de Patologia e Imunologia Molecular da Universidade do Porto. ${ }^{6}$

É possível observar que o crescimento exponencial da MN tem estimulado a busca pelo conhecimento de seus efeitos e impactos, promovendo o aumento de pesquisas que procurem dar respostas a esses questionamentos. A modalidade qualitativa foi a mais prevalente nos artigos analisados mostrando-se, de fato, mais compatível e coerente com a proposta e a filosofia da MN, uma vez que consideram o discurso e a narrativa dos sujeitos participantes de grande valor para a análise dos resultados. No entanto, não podemos desconsiderar que os estudos apoiados em metodologias quantitativas, ou mistas, também trouxeram resultados significativos.

6 Ana Luiza Novis, Fátima Geovanini, and Lorraine Veran. Medicina Narrativa: A Arte Do Encontro. 1st ed. (Rio de Janeiro: Thieme Revinter, 2021). 
Estudos voltados para habilidades de comunicação, escuta e, com maior relevância, empatia, mostraram-se os mais recorrentes. A promoção da empatia e o desenvolvimento das habilidades de comunicação, têm sido usualmente associados à prática da MN. Reafirmando essa relação, os resultados apresentados nos artigos, que se propuseram a avaliar o impacto desses aspectos, mostraram-se bastante positivos. Segundo a literatura, os grupos de estudantes com alta empatia, desde a entrada na graduação de medicina, tendiam à queda dessa qualidade durante o curso enquanto os com baixa capacidade empática tendiam ao crescimento, sendo o nível de empatia mais sensível à mudança do que as habilidades de comunicação. ${ }^{7}$

Vários instrumentos têm sido desenvolvidos com o objetivo de medir a empatia, em sua maioria por autorrelato, sendo eles The Hogan Empathy Scale, Adjective Check List Empathy Scale, Jefferson Scale of Empathy. Alguns deles são aplicáveis ao contexto médico, sendo o último o mais encontrado em estudos sobre a aplicação da Medicina Narrativa. ${ }^{8,9}$

A despeito de um certo otimismo, que pode contaminar a análise dos dados, visto que provavelmente todos esses pesquisadores já se mostravam previamente favoráveis à $\mathrm{MN}$, não foram desconsideradas as principais condições para o alcance dos seus objetivos, a saber: o participante estar aberto e favorável à realização das atividades e à continuidade do trabalho, visto que a prática diária das atividades clínicas pode contribuir para o declínio das habilidades emocionais e, consequentemente, relacionais. Um artigo destacou que ter resultados positivos, ao final das atividades de MN, não podem garantir uma boa prática clínica. Os resultados das duas pesquisas, que identificam um aumento nas pontuações nos exames referentes às avalições médicas e técnicas globais, desassociadas da relação médico-paciente, podem estar relacionados a um aumento de habilidades de interpretação textual, mais interesse pelo conteúdo, por menores taxas de burnout, entre outros. Pesquisas que aprofundem os motivos para essa melhora no desempenho acadêmico devem ser desenvolvidas no futuro. $^{10}$

Para uma prática médica consciente, os processos de reflexão na ação e a experiência afetiva, que elabore um aprendizado duradouro são essenciais. A reflexão pode estimular a empatia e a sabedoria prática, orientando profissionais de saúde na clínica. A atitude reflexiva está associada ao uso

7 Natália Medeiros, Thaís Santos, Eliana Trindade, and Karlo Almeida. Avaliação do desenvolvimento de competências afetivas e empáticas do futuro médico. Revista Brasileira de Educação Médica 37, 4 (2013): 515-525.

8 Darryl Cross, and C. F. Sharpley. "Measurement Of Empathy With The Hogan Empathy Scale". Psychological Reports 50, 1 (1982): 62-62.

9 Barbara May, and Martha R. Alligood. "Basic Empathy In Older Adults: Conceptualization, Measurement, And Application". Issues In Mental Health Nursing 21, 4 (2000): 375-386.

10 Arntfield, Shannon L., Kristen Slesar, Jennifer Dickson, and Rita Charon. 2013. "Narrative Medicine As A Means Of Training Medical Students Toward Residency Competencies". Patient Education And Counseling 91 (3): $280-286$. 
eficaz de feedback na educação médica e o valor de treinar professores como "treinadores reflexivos" tem sido muito enfatizado. A reflexão não é necessariamente intuitiva para alunos ou professores, portanto, desenvolvedores de programas de graduação são desafiados a conceber uma pedagogia inovadora que promova a capacidade reflexiva. As humanidades, as artes em geral e a literatura e as narrativas, em particular, servem como recursos valiosos para promover a reflexão. Sob o guardachuva da medicina narrativa, o estudo da literatura e a escrita reflexiva podem promover a reflexão na atenção médica, construir habilidades de observação e interpretação clínica e fortalecer a atenção empática aos pacientes, desenvolvendo a competência narrativa necessária à prática clínica. ${ }^{11,12,13,14}$

Dentre os demais fatores analisados, houve destaque também para a gestão das emoções e o autocuidado, embora apenas dois tenham citado o burnout, que é tão diretamente relacionado ao cuidado - do outro, do paciente, da equipe - um dos principais objetivos da MN. Nenhuma pesquisa buscou os impactos na criatividade, ainda que Charon argumente que a segurança em grupos melhora a confiança e a criatividade e outros estudos afirmem que a segurança psicológica pode facilitar a adesão de comportamentos essenciais, como solicitar ajuda ou assumir erros. Isso aponta para o potencial da arte e da literatura como ferramentas capazes de democratizar relações e suas posições hierárquicas, contribuindo para a qualidade das relações multiprofissionais. ${ }^{15,16}$

Um único artigo identificou, através de grupo controle, melhor eficácia nos resultados quando foi possível associar a prática aos estudos teóricos. ${ }^{17}$ Considera-se esse dado relevante, podendo contribuir significativamente para a elaboração de novas propostas no campo da MN. Diversos cursos de graduação médica, de Universidades do Rio de Janeiro - UNESA, UERJ, UFRJ e a Faculdade Souza Marques - vêm incluindo disciplinas, optativas ou obrigatórias, cuja metodologia se baseia na MN. Representantes dessas instituições têm trabalhado de forma pioneira, com o intuito de oferecer aos alunos uma formação ampliada, desenvolvendo habilidades de comunicação, empatia, escuta, postura reflexiva e competência narrativa contribuindo para que esses estudantes possam, ainda durante a

11 Howard S.Schwartz, and Donald A. Schon. "The Reflective Practitioner: How Professionals Think In Action.". Administrative Science Quarterly 32, 4 (1987): 614.

12 Robert G Bing-You. "Why Medical Educators May Be Failing At Feedback". JAMA 302, 12 (2009): 1330. doi:10.1001/jama.2009.1393.

13 Melissa Nothnagle, Roberta Goldman, Mark Quirk, and Shmuel Reis. "Promoting Self-Directed Learning Skills In Residency: A Case Study In Program Development". Academic Medicine 85, 12 (2010): 1874-1879.

14 Paul George, Shmuel Reis, Margaret Dobson, and Melissa Nothnagle. "Using A Learning Coach To Develop Family Medicine Residents' Goal-Setting And Reflection Skills". Journal Of Graduate Medical Education 5, 2 (2013): $289-293$. 15 Rita Charon, Nellie Hermann, and Michael J. Devlin. "Close Reading And Creative Writing In Clinical Education". Academic Medicine 91, 3 (2016): 345-350.

16 Amy C Edmondson, Monica Higgins, Sara Singer, and Jennie Weiner. "Understanding Psychological Safety In Health Care And Education Organizations: A Comparative Perspective". Research In Human Development 13, 1 (2016): $65-83$. 17 Barbara May, and Martha R. Alligood. "Basic Empathy In Older Adults: Conceptualization, Measurement, And Application". Issues In Mental Health Nursing 21, 4 (2000): 375-386. 
graduação, associar os ensinamentos da $\mathrm{MN}$ às demais disciplinas cursadas, como por exemplo, às aulas de propedêutica onde aprendem o modelo tradicional de anamnese. Estendendo a atuação para além do território formal das universidades e, com o objetivo de propagar a MN na educação em saúde, teve início em maio de 2020 um Grupo de Estudos online, via plataforma zoom, agregando profissionais, docentes e discentes de todo o Brasil, contando ainda com a participação e apoio de representantes de Portugal - Universidade de Lisboa e Universidade do Porto. Além disso, o grupo foi contemplado com a participação especial da própria precursora, a médica e literata Rita Charon.

\section{Conclusão}

O crescimento exponencial das humanidades médicas e da Medicina Narrativa traz uma forte esperança para o campo da Educação Médica. Esperança por uma formação ampla, onde a arte possa contribuir para diminuir a aridez da medicina enquanto ciência, valorizando a medicina enquanto arte. Esperança por médicos que ouçam as histórias dos pacientes e possam valorizá-las, dando assim destaque ao doente e não exclusivamente à doença. Esperança por médicos com competência narrativa para que consigam ser tocados e agir em prol do cuidado - dele próprio, do paciente e seus familiares e da sua equipe.

Embora desde o século XIX a literatura, e as artes em geral, já tenham sido incluídas nas escolas médicas, sabemos que a MN, enquanto proposta metodológica, mostra-se ainda um campo recente. Com sua crescente e forte atuação acreditamos que muitas pesquisas que busquem identificar a sua eficácia devam ainda ser desenvolvidas. Neste estudo identificamos um número exponencial desses estudos, sendo a maioria deles de base qualitativa, apenas quatro foram quantitativos e um quali-quantitativo. Importante destacar que consideramos legítimo o intuito de conhecer melhor a MN, porém achamos que este não deve justificar a tentativa de encaixar a MN em avaliações exclusivamente quantitativas, tal como acontece nas demais áreas no campo da saúde. Se optamos e estimulamos a escuta das histórias, das vozes de nossos pacientes, porque não considerar e valorizar o discurso dos participantes em nossas atividades de formação e pesquisa?

A MN pode oferecer um forte potencial transformador. No entanto, muitas das vezes as transformações provocadas não serão necessariamente mensuráveis quantitativamente. No que ela atua pode ser invisível aos números, mas não escapará ao discurso, à escuta de uma história, em suas diversas formas narrativas. Afinal, somos seres históricos, vivemos e existimos na história e pelas histórias. 


\section{Referências Bibliográficas}

Afanador, Amaya. Simulación clínica y aprendizaje emocional. Revista Colombiana de Psiquiatría 41, (2012):44-51.

Almeida, Hélcia, et al. Desenvolvendo competências em comunicação: uma experiência com a medicina narrativa. Revista Brasileira de Educação Médica 29, 3 (2020):208-216.

Alves, Paulo Cesar. The Death of Ivan Ilyich and multiple dimensions of illness. Ciência \& Saúde Coletiva, Rio de Janeiro, 23, 2 (2018).

Appalasamy, Jamuna Rani et al. The effectiveness of culturally tailored video narratives on medication understanding and use self-efficacy among stroke patients: A randomized controlled trial study protocol. Medicine 97, 22 (2018):10876.

Aragão, Júlio Cesar Soares, et al. A jornada do acadêmico de Medicina - Um modelo simbólico da formação Médica. Revista Brasileira de Educação Médica 42, 1 (2018):40-46.

Arntfield, Shannon, et al. Narrative medicine as a means of training medical students toward residency competencies. Patient Education and Counseling 91, 3(2013):280-286.

Baines, Rebecca, et al. 2018. The impact of patient feedback on the medical performance of qualified doctors: a systematic review. BMC Medical Education 18, 1 (2018):173.

Bastos, Daniela, Antônio Cunha and Alicia de Souza. The experience of family medicine interns conducting McGill ILLNESS narrative interview with non-compliant chronic patients. Revista Brasileira de Educação Médica, 42, 3 (2018):178-188.

Bello, Alejandra, and Volnei Garrafa. Crítica à instituição médica moderno-industrial a partir do microssistema linguístico de Ivan Illich. Saúde em Debate, 42, 116 (2018):263-273.

Benedetto, Maria Auxiliadora and Dante Gallian. Narrativas de estudantes de medicina e enfermagem: currículo oculto e desumanização em saúde. Interface - Comunicação, Saúde, Educação 22, 67 (2018):1197-1207.

Bing-You, Robert. Why medical educators may be failing at feedback. JAMA 302, 12 (2019):1330.

Bokhour, Bárbara G., et al. Can stories influence African-American patients' intentions to change hypertension management behaviors? A randomized control trial. Patient Education and Counseling 99, 9 (2016):1482-1488.

Callwood, Alison, et al. Personal domains assessed in multiple mini interviews (MMIs) for healthcare student selection: A narrative synthesis systematic review. Nurse Education Today 64, (2018): $56-64$.

Cardoso, Maria Helena Cabral de Almeida. História e medicina: a herança arcaica de um paradigma. História, Ciências, Saúde-Manguinhos 6, 3 (2000):551-575.

Castro, José Renato, et al. Abordagens de ensino e pesquisa na pós-graduação em saúde: da realidade da disciplina a "utopia" transdisciplinar. Interface - Comunicação, Saúde, Educação 15, 39 (2011):1025

Centanaro,Meza and Gabriel Adolfo. Enfoque holístico al paciente neurológico: el aporte de Oliver Sacks. Acta Neurológica Colombiana 31, 3(2015):342-349.

Charon, Rita, Nellie Hermann and Michael J Devlin. Close reading and creative writing in clinical education. Academic Medicine 91, 3 (2016):345-350. 
Charon, Rita, et al. The principles and practice of narrative medicine. Nova York, NY: Oxford University Press, 2017.

Chen, Po-Jui, Chien-Da Hung and San-Jou Yeh. Impact of a narrative medicine programme on healthcare providers' empathy scores over time. BMC Medical Education 17, 1 (2017).

Chunming, Wang Michael, et al. Burnout in medical students: a systematic review of experiences in Chinese medical schools. BMC Medical Education 17, 1 (2017):217.

Claro, Lenita and Anna Alice Mendes. Uma experiência do uso de narrativas na formação de estudantes de Medicina. Interface - Comunicação, Saúde, Educação 22, 65 (2018):621-630.

Colares, Karla T. Pereira, and Wellington de Oliveira. Metodologias ativas na formação profissional em saúde: uma revisão. Sustinere - Revista de Saude e Educação 6, 2(2018):300.

Constantin, Francismo Gelman. Poesia e humanidades médicas no Cone Sul: algumas proposições a partir da obra de Virna Teixeira. Physis, Rio de Janeiro, 29, 4(2019).

Cordeiro, Alexander Margno, et al. Revisão sistemática: uma revisão narrativa. Revista do Colégio Brasileiro de Cirurgiões, 34, 6(2007):428-431.

Cordeiro, Franciele Roberta, and Maria Henriqueta Luce Kruse. A produção do curriculo do final da vida por meio do dispositivo pedagógico da mídia. Interface - Comunicação, Saúde, Educação 19, 55(2015):1193.

Coret, Alon, et al. Patient narratives as a teaching tool: a pilot study of first-year medical students and patient educators affected by intellectual/developmental disabilities. Teaching and Learning in Medicine 30, 3(2018):317-327.

Cross, Darryl, et al.. Measurement of empathy with the Hogan Empathy Scale. Psychological Reports 50, 1(1982):62.

Daryazadeh, Saeideh, et al. Impact of a narrative medicine program on reflective capacity and empathy of medical students in Iran. Journal of Educational Evaluation for Health Professions, 17(2020):3.

Davenport, Nancy H M. Medical residents use of narrative templates in storytelling and diagnosis. Soc Sci Med 73, 6(2011):873-881.

De Araújo Júnior, José Sebastião, et al. O ensino de anatomia humana no contexto da educação médica: uma retrospectiva histórica. Research, Society and Development 9, 7(2020):958975173958975173.

De Oliveira, Filipe, et al. A experiencia dos diários reflexivos no processo formativo de uma residência multiprofissional em saúde da família. Interface - Comunicação Saúde Educação 17, 44 (2013):201.

Dobler, Claudia, et al. Impact of decision aids used during clinical encounters on clinician outcomes and consultation length: a systematic review. BMJ Quality \& Safety 28, 6(2019):499-510.

Dunker, Christian Ingo Lenz. Discontent, suffering and symptom: a Lacanian diagnostic re-reading via animist perspectivism. Tempo Social, São Paulo, 23, 1(2011):115-136.

Edmondson, Amy, et al. Understanding psychological safety in health care and education organizations: A comparative perspective. Research in Human Development 13, 1(2016):65-83.

Farquhar, Morag, et al. What are the key elements of educational interventions for lay carers of patients with advanced disease? A systematic literature search and narrative review of structural 
components, processes and modes of delivery. Journal of Pain and Symptom Management 52, 1(2016):117-130.

Favoreto, Cesar Augusto Orazem, and Cristiane Coelho Cabral. Narrativas sobre o processo saúdedoença: experiências em grupos operativos de educação em saúde. Interface - Comunicação, Saúde, Educação 13, 28(2009):7-18.

Favoreto, César Augusto, and Kenneth Rochel de Camargo Jr, et al. La narrativa como herramienta para el desarrollo de la práctica clínica. Interface - Comunicação, Saúde, Educação 15, 37 (2011):473-483.

Fernandes, Isabel. A pertinência da Medicina Narrativa na prática clínica. Revista Portuguesa de Medicina Geral e Familiar 30, 5(2014): 289-290.

Fernandes, Isabel. Leituras holísticas: de Tchékhov à Medicina Narrativa. Interface - Comunicação, Saúde, Educação 19, 52(2015):71-82.

Fonsêca, Graciela Soares, and João Victor Garcia de Souza. A narrativa de um percurso formativo: (re) significando a formação médica. Interface - Comunicação, Saúde, Educação, 23 (2019). Doi: https://doi.org/10.1590/Interface.180059

George, Paul, Shmuel Reis, Margaret Dobson and Melissa Nothnagle. Using a learning coach to develop family medicine residents' goal-setting and reflection skills. Journal of Graduate Medical Education 5, 2(2013):289-293.

Gillis, Amy, Marie C Morris, and Paul F Ridway. Communication skills assessment in the final postgraduate years to established practice: a systematic review. Postgrad Med $J$ 91, 1071(2015):13-21.

Ginsburg, Shiphra, et al. Reading between the lines: faculty interpretations of narrative evaluation comments. Medical Education 49, 3(2015):296-306

Gomes, Romeu and Valeria Vernaschi Lima. Narratives on educational processes in health. Ciência \& Saúde Coletiva 24, 12(2019):4687.

Gorniewicz, James, et al. Breaking bad news to patients with cancer: A randomized control trial of a brief communication skills training module incorporating the stories and preferences of actual patients. Patient Education and Counseling 100, 4(2017):655-666.

Goupy, François, et al. L'enseignement de la médecine narrative peut-il être une réponse à l'attente de formation des étudiants à la relation médecin-malade? La Presse Médicale 42, 1(2013):18 .

Gowda, Deepthiman. et al. Implementing an interprofessional narrative medicine program in academic clinics: feasibility and program evaluation. Perspectives on Medical Education 8, 1(2019):52-59.

Grossman, Eloísa, and Maria Helena Cabral de Almeida Cardoso. As narrativas em medicina: contribuições à prática clínica e ao ensino médico. Revista Brasileira de Educação Médica 30, 1(2006):6-14.

Harrison, Madaline B., and Nicole Chiota-Mccollum. Education Research: an arts-based curriculum for neurology residents. Neurology 92, 8(2019):879-883.

Hatala, Rose, et al. Using in-training evaluation report (ITER) qualitative comments to assess medical students and residents: a systematic review. Academic Medicine 92, 6(2017):868-879. 
Ianni, Aurea Maria Zollner. The thematic field of the social sciences in health in Brazil. Tempo Social, São Paulo, 27, 1(2015):13-32.

Jha, Vikram, et al. Patients as teachers: a randomised controlled trial on the use of personal stories of harm to raise awareness of patient safety for doctors in training. BMJ Quality \& Safety 24, 1(2014):21-30.

Jiménez, Rosa C. A. Medicina narrativa: el paciente como "texto", objeto y sujeto de la compasión. Acta Bioethica 23, 2(2017):351-359.

Johna, Samir, Brandon Woodward, and Sunal Patel. 2014. What can we learn from narratives in medical education? Perm J 18, 2(2014):92-94.

Juárez, Lorenzo, et al. Cuidados basados en narrativas: redefiniendo la jerarquía de la evidencia. Index de Enfermería 22, 2(2013):55-59.

Junior, Helvo, Laura Feuerwerker and Marcelo Land. Educação em saúde ou projeto terapêutico compartilhado? O cuidado extravasa a dimensão pedagógica. Ciência \& Saúde Coletiva 20, 2(2015):537.

Khorana, Alok, Micheelle Shayne and David N Korones. Can literature enhance oncology training? A pilot humanities curriculum. Journal of Clinical Oncology 29, 4(2011):468-471.

Konttila, Jenni, et al. Healthcare professionals' competence in digitalisation: A systematic review. Journal of Clinical Nursing 28, 5-6(2019):745-761.

Kottow, Miguel ¿Bioética narrativa o narrativa bioética? Revista Latino -Americana de Bioética 16, 2(2016):58-69.

Kumagai, Arno K. Perspective: acts of interpretation. A philosophical approach to using creative arts in medical education. Academic Medicine 87, 8(2012):1138-1144.

Lekhtsier, Vitaly, and Anna Gotlib. Medicina narrativa enfocada a la investigación empírica social: el contexto ruso. Salud Colectiva 13, 2(2017):239-252.

Marco-Gómez, Bárbara, et al. O processo de adoecimento dos médicos e as suas consequências acerca do profissionalismo. Acta Bioethica 24, 2(2018):199-210.

Marin, Maria José, et al. Ensino em saúde: aprendizagem a partir da prática profissional. Ciência \& Saúde Coletiva, 24, 5(2019). Doi: https://doi.org/10.1590/1413-81232018245.04412019

Maudsley, Gillian, and David Taylor. Analysing synthesis of evidence in a systematic review in health professions education: observations on struggling beyond Kirkpatrick. Medical Education 25, 1(2020):1731278.

May, Barbara, and Martha R. Alligood. Basic empathy in older adults: conceptualization, measurement, and application. Issues in Mental Health Nursing 21, 4(2000):375-386.

Medeiros, Natália, et al. Avaliação do desenvolvimento de competências afetivas e empáticas do futuro médico. Revista Brasileira de Educação Médica 37, 4(2013):515-525.

Meisel, Zachary F. and Jason Karlawish. Narrative vs evidence-based medicine: and, not or. JAMA 306, 18(2011):2022-2023.

Mello, Carolina, et al. Metodologias de ensino e formação na area da saúde: revisão de literatura. Revista CEFAC: Atualização Cientifica em Fonoaudiologia e Educação 16, 6(2014):2015. 
Moon, Margareth, et al. Analyzing reflective narratives to assess the ethical reasoning of pediatric residents. Narrat Inq Bioeth 3, 2(2013):165-174.

Moore, M. Moore, et al. Communication skills training for healthcare professionals working with people who have cancer. The Cochrane database of systematic reviews 7, 7(2018):CD003751.

Nicklas, Daniel, et al. Using Digital Stories to Reflect on the Culture of Overuse, Misuse, and Underuse in Medicine and Enhance the Patient-Provider Relationship. Acad Pediatr 17, 6(2017):694-696.

Nothnagle, Melissa. et al. Promoting self-directed learning skills in residency: A case study in program development. Academic Medicine 85, 12(2010):1874-1879.

Novis, Ana Luiza, Fátima Geovanini and Lorraine Veran. Medicina Narrativa: a arte do encontro. 1. ed. Rio de Janeiro: Thieme Revinter, 2021.

Nunes, Everardo Duarte. A sociologia da saúde no Brasil: a construção de uma identidade. Ciência \& Saúde Coletiva 19, 4(2014):1041.

O’Mahony, Seamus. Against narrative medicine. Perspect Biol Med, vol. 56, 4(2013):611-619.

O'Mara-Eves, Alison, et al. Using text mining for study identification in systematic reviews: a systematic review of current approaches. Systematic reviews 4, 1(2015):5.

Oliveira, Claudia Maria, Nildo Alves Batista, Sylvia Helena Batista, Lúcia Uchôa-Figueiredo. A escrita de narrativas e o desenvolvimento de práticas colaborativas para o trabalho em equipe. Interface - Comunicação, Saúde, Educação 20, 59(2016):1005-1014.

Oshman, D Lauren, and Gene N Combs. Integrating motivational interviewing and narrative therapy to teach behavior change to family medicine resident physicians. Int J Psychiatry Med, 51, 4(2016):367-378.

Palácio, Maria Augusta Vasconcelos, Laís Barreto Gonçalves, and Miriam Struchiner. A narrativa do aluno de medicina na formação em atenção primária à saúde: potencializando espaços de aprendizagem mediados pelas tecnologias digitais. Revista Brasileira de Educação Médica 43, 1(2020):330-340.

Pastor Junior, Américo, et al. Recepção audiovisual na educação médica: leituras de um vídeo educativo de psicologia médica por estudantes de medicina. Revista Brasileira de Educação Médica 36, 4(2012):516-523.

Pelgim, Elisabeth A. M, et al. Quality of written narrative feedback and reflection in a modified miniclinical evaluation exercise: an observational study. BMC Medical Education, 12, (2012):97.

Peña Lora, Marianela Rosa. La Narración oral: Alternativa para el desarrollo de una experiencia educativa con perspectivas de género. Revista História de la Educación Latinoamericana 17, 24(2015):73-92.

Polly, Wc Li, and Doris Sf Yu. A modeling-based narrative intervention to promote timely careseeking in patients with acute myocardial infarction: A pilot randomized controlled trial and feasibility analysis. European Journal Of Cardiovascular Nursing: Journal Of The Working Group on Cardiovascular Nursing of the European Society of Cardiology 18, 3(2019):215223.

Quaintance, Jennifer L., Louise Arnold and George S Thompson. What students learn about professionalism from faculty stories: an appreciative inquiry approach. Academic Medicine 85, 1(2010):118-123. 
Ramis Olivos, Álvaro. Bioética narrativa y salud intercultural mapuche: Aportes a su fundamentación y complementación. Veritas 34(2016):93-116.

Rian, Johanna, and Rachel Hammer. The practical application of narrative medicine at Mayo Clinic: imagining the scaffold of a worthy house. Cult Med Psychiatry 37, 4(2013):670-680.

Rivlin, Katherine and Carolyn L. Westhoff. Navigating uncertainty: narrative medicine in pregnancy options counseling education. Patient Education and Counseling 102, 3(2019):536-541.

Rocha, Sheyla Ribeiro, et al. Avaliação de Habilidades de Comunicação em Ambiente Simulado na Formação Médica: Conceitos, Desafios e Possibilidades. Revista Brasileira de Educação Médica, 43, 1(2020):236-245.

Rosário, Francisco S. Anamnese Versus História de Vida na Compreensão do Sofrimento do Outro. Medicina Interna 25, 3(2018):233-236.

Ross, David A, et al. A narrative-based approach to teaching diagnostic criteria. Acad Psychiatry 38, 6(2014):706-708.

Rothlind, Erica, et al. The informal curriculum of family medicine - what does it entail and how is it taught to residents? A systematic review. BMC Family Practice 21, 1(2020):49.

Rowland, Paula, et al. Patient involvement in health professionals' education: a meta-narrative review. Advances in Health Sciences Education: Theory and Practice 24, 3(2019):595-617.

Sánchez Mendiola, Melchor. Liderazgo en medicina: ¿debemos enseñarlo y evaluarlo? Investigación en Educación Médica, 4, 14(2015):99-107.

Santillán-Doherty, Patricio. Medicina, narrativa y ser. Neumología y Cirugía de Tórax 73, 2(2014):102-105.

Sarangi, Srikannt. Reconfigurando self/identidade/status/papel: o caso do desempenho de papel profissional em encontros na área da saúde. Veredas - Revista de Estudos Linguísticos 18, 2(2014):321.

Schon, Donald, et al. The reflective practitioner: how professionals think in action. Administrative Science Quarterly 32, 4(1987):614.

Sevalho, Gil. O conceito de vulnerabilidade e a educação em saúde fundamentada em Paulo Freire. Interface - Comunicação Saúde Educação 22, 64(2018):177.

Silva, Georgia. A construção do ser médico e a morte: significados e implicações para a humanização do cuidado. Serviço de Biblioteca e Documentação da Faculdade de Medicina da Universidade de São Paulo, São Paulo, 2006.

Soares Brandão, Carolina et al. Centros de simulação e projeto pedagógico: dois lados da mesma moeda. Scientia Médica 28, 1(2018)

Soares, Márcia Oliveira Mayo. Anamnesis impact to an integrated care: Portuguese students' view. Revista Brasileira em Promoção da Saúde, Fortaleza, 29, (2016):66-75.

Sousa, Islândia, et al. Medicina Tradicional Complementar e Integrativa: desafios para construir um modelo de avaliação do cuidado Ciência \& Saúde Coletiva 23, 10(2018):3403-3412.

Steinert, Yvonne, Laura Naismith, and Karen Mann. Faculty development initiatives designed to promote leadership in medical education. A BEME systematic review: BEME Guide No. 19. Medical Teacher 34, 6(2012):483-503. 
Stelet, Bruno Pereira, et al. 2017. Portfólio Reflexivo: subsídios filosóficos para uma práxis narrativa no ensino médico. Interface - Comunicação, Saúde, Educação 21, 60(2017):165-176.

Stelet, Bruno Pereira, et al. Portfólio Reflexivo: subsídios filosóficos para uma práxis narrativa no ensino médico. Interface - Comunicação, Saúde, Educação 21, 60(2016):165-176.

Thistlethwaite, Jill Elizabeth, et al. The effectiveness of case-based learning in health professional education. A BEME systematic review: BEME Guide No. 23. Medical Teacher 34, 6(2012):421-444.

Urday-Fernández, Dayanara, and María Sofia Cuba-Fuentes. Medicina narrativa. Anales de la Facultad de Medicina 80, 1(2019):109-113.

Val, Alexandre Costa, et al. Psicanálise e Saúde Coletiva: aproximações e possibilidades de contribuições. Physis 27, 4(2017):1287-1307.

Van-Beusekom, Mara, et al. Communication skills training for the radiotherapy team to manage cancer patients' emotional concerns: a systematic review. BMJ Open 9, 4(2019):025420.

Vosgerau, Dilmeire and Joana Paulin Romanowski. Estudos de revisão: implicações conceituais e metodológicas. Revista Diálogo Educacional 14, 41(2014):165.

Wesley, Tiffany, Diana Hamer and George Karam. Implementing a narrative medicine curriculum during the internship year: an internal medicine residency program experience. The Permanente Journal, 2018.

Yang, Ningxi, et al. Does narrative medicine education improve nursing students' empathic abilities and academic achievement? A randomised controlled trial. Journal of International Medical Research 46, 8(2018):3306-3317.

Yeates, Peter et al. Relatively speaking: contrast effects influence assessors' scores and narrative feedback. Medical Education 49, 9(2015):909-919. 